\title{
Prediction of Basketball Competition Scores Based on BP Neural Network Algorithm
}

\author{
Yong-Sheng WANG ${ }^{1, a}$, Quan-Ying $\mathrm{LI}^{2, a}$ \\ ${ }^{1}$ Department of Physical education, North-west University, Xi'an, 710069, China \\ ${ }^{2} \mathrm{Xi}$ 'an electric power college, Xi'an, 710032, China \\ a724350372@qq.com
}

Keywords: Neural Network, BP, Basketball Competition, Competition Scores.

\begin{abstract}
In the thesis, we use the time sequence of basketball competition as the statistical data to predict the technical statistical indicators for the basketball team based on BP neural network algorithm. Our study aims at discussing the availability and applications of the BP neural network in the score prediction.
\end{abstract}

\section{Introduction}

Prediction of competition scores is the prediction of the future sports scores based on the existing sport scores. Scientific prediction of competitions can not only provide athletes and coaches with certain targets for training and competitions, but also track and judge the tendency and regulations of sport items. Therefore, the prediction of competition scores has increasingly become crucial in scientific sport managements and competitions. Also, it has been concerned by the leaders, managers and researchers during current days.

With the development of dynamic system theories, artificial neural networks (ANNs) and expert system, sports predictors start to use better approaches that suits for human mind and the dynamic changes of predictive environments. ANNs can learn existing data automatically without too much complex processes and get close to the best function that can classify the samples [1]. Artificial expert system can gather the knowledge and experiences of human and give quantitative results, which makes the problems be more close to actual issues. Therefore, ANNs and artificial intelligence have successfully extend the potential development of predictive systems [2]. Here, we use the time sequence of basketball competition as the statistical data to predict the technical statistical indicators for the basketball team based on BP neural network algorithm. Our study aims at discussing the availability and applications of the BP neural network in the score prediction.

\section{Algorithm and Model Development of BP Neural Network}

\section{BP Neural Network}

BP neural network is the feed-forward neural network with the transmission of errors. It is usually made up of the input, output and several hidden layers. Each layer consists of a series of nodes, which represents the neurons [3]. The upper layer connects with the utter layer via the weights. Different layers interconnect with each other and there is no connection among neurons that in the same layer. A typical BP network has three layers, including one hidden layer, which is shown in Figure 1:

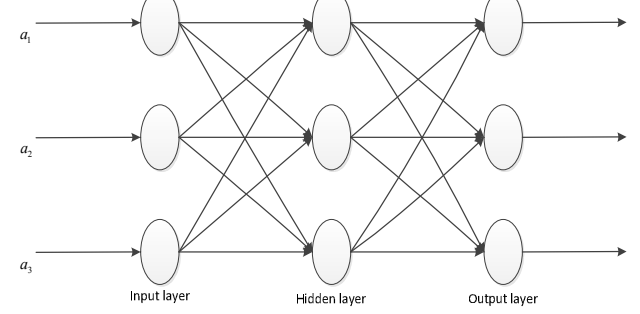

Fig. 1 BP Network Structure 


\section{Model Development of BP Neural Network}

BP network is based on the transmission of errors using related algorithms. The learning processes consist of four processes: the input mode transmitted from the input layer to the output layer; the transmission of error signal of the desired output and actual output via the connected weights; the cross-linked " training memory" of "mode transmission" and "error transmission"; the total generalization of "learning convergence" of the network[4]. The algorithm process is shown in Figure 2:

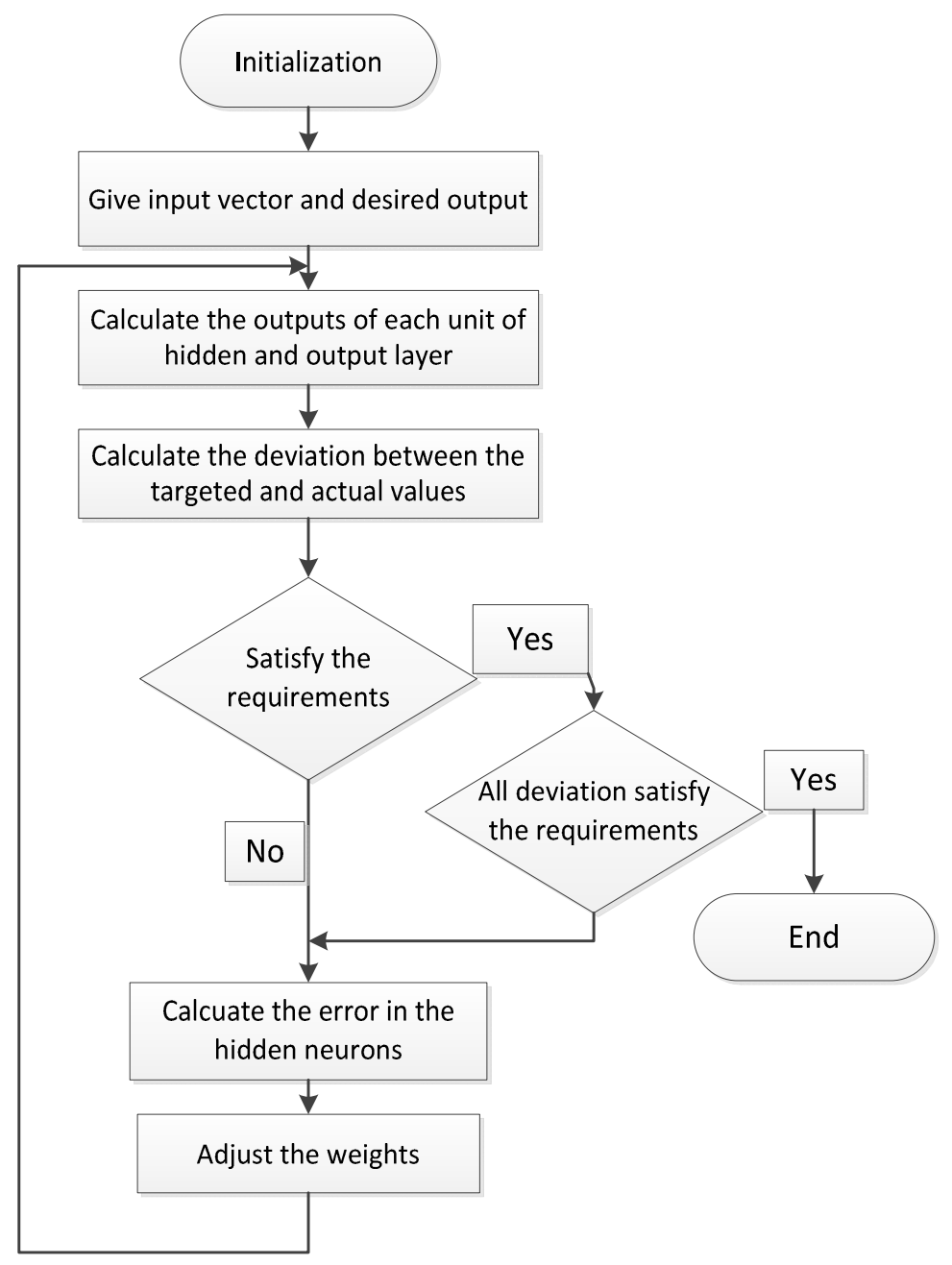

Fig. 2 The learning algorithm of BP neural network.

In Matlab neural network toolbox, we can find the improved algorithm of BP neural network. It provides us with enough orders and functions for modeling, which can simplify the difficulties and steps when modeling[5]. In this article, we use the neural network toolbox of Matlab to develop the predictive model for basketball technical statistics.

\section{Model Development for Predicting the Basketball Competition Scores using ANNs}

\section{Predictive Model Development Approaches of ANNs}

The ANN model for basketball competition scores is the method based on data. It means that using ANNs can generate a non-linear fitting process to get close to a time sequence or a similar time sequence. The future time values can be expressed utilizing the past time values via the clear logical relationship of ANNs.

Predictive ANNs can be divided into two kinds of predictions, the single-variable prediction based on time sequence and the multi-variables prediction based on time sequence. In terms of the 
single-variable prediction, we can set the time sequence as $\left\{X_{i}\right\}$, where $X_{n}, X_{n+1}, \cdots, X_{n+m}$. The prediction at the future $n+m+k(K>0)$ is the prediction of the value of $X_{n+m+k}$. That is to say, it draws the non-linear relationship of the historic data $X_{n}, X_{n+1}, \cdots, X_{n+m}$ and $X_{n+m+K}$. In terms of the multi-variables prediction, we set the time sequence as $\left(X_{1 n}, X_{2 n}, \cdots, X_{i n}\right),\left(X_{1 n+1}, X_{2 n+1}, \cdots, X_{i n+1}\right), \cdots$, $\left(X_{1 n+m}, X_{2 n+m}, \cdots, X_{i n+m}\right)$. We can predict the future values at $n+m+k(k>0)$. Being similar to the single-variable prediction, the ANNs can fit the non-linear relationship $X_{n+m+K}=F\left\{\left(X_{1 n}, X_{2 n}, \cdots\right.\right.$, $\left.\left.X_{\text {in }}\right),\left(X_{1 n+1}, X_{2 n+1}, \cdots, X_{i n+1}\right) \cdots,\left(X_{1 n+m}, X_{2 n+m}, \cdots, X_{i n+m}\right)\right\}$.

Rolling prediction, the so-called iterated one-step prediction. We can do a single-step prediction, and give the feedback of output to the input layer, which can be used for predicting the values at k kinds of times.

$\begin{array}{lcc}\text { Step } & \text { The neural network input } & \text { Output (prediction) } \\ 1 & \mathrm{X}_{\mathrm{n}}, \mathrm{X}_{\mathrm{n}+1}, \cdots, \mathrm{X}_{\mathrm{n}+\mathrm{m}} & \mathrm{X}_{\mathrm{n}+\mathrm{m}+1} \\ 2 & \mathrm{X}_{\mathrm{n}+1}, \mathrm{X}_{\mathrm{n}+2}, \cdots, \mathrm{X}_{\mathrm{n}+\mathrm{m}+1} & \mathrm{X}_{\mathrm{n}+\mathrm{m}+2} \\ \cdots & \cdots & \cdots \\ \mathrm{K} & \mathrm{X}_{\mathrm{n}+\mathrm{k}-1}, \mathrm{X}_{\mathrm{n}+\mathrm{k}}, \cdots, \mathrm{X}_{\mathrm{n}+\mathrm{m}-1} & \mathrm{X}_{\mathrm{n}+\mathrm{m}+\mathrm{k}}\end{array}$

\section{The Achievement of Rolling Prediction}

Here we take a technical statistical results of a university basketball team to illustrate the approach of rolling prediction using ANNs. We collected five competition data in recent four years as the training sample. According to the existing mean shooting percentage and the actual situations, we can set the shooting percentage of the input variable of the model. The statistical data is shown as follows (table 1):

Table 1 Shooting a game

\begin{tabular}{ccccc}
\hline & 1 & 2 & 3 & 4 \\
\hline The first group & 0.4328 & 0.4225 & 0.4252 & 0.4082 \\
The second group & 0.4612 & 0.4116 & 0.3611 & 0.4638 \\
The third group & 0.3884 & 0.4517 & 0.4628 & 0.4326 \\
The fourth group & 0.4371 & 0.4103 & 0.4215 & 0.4597 \\
The fifth group & 0.4296 & 0.4908 & 0.4318 & 0.4257 \\
The Sixth group & 0.3915 & 0.5121 & 0.4459 & 0.4118 \\
\hline
\end{tabular}

To make the weights of networks are in the range of [0,1], we must do normalization of the inputted data, using the equation shown as follows:

$$
S_{i}=\frac{x_{i}-x_{\min }}{x_{\max }-x_{\min }}
$$

where $x_{\max }, x_{\min }$ are the maximum and minimum of original data respectively. The processed data is shown as follows (table 2):

Table 2 The unitary data of shooting a game

\begin{tabular}{ccccc}
\hline & 1 & 2 & 3 & 4 \\
\hline The first group & 0.4328 & 0.4225 & 0.4252 & 0.4082 \\
The second group & 0.4612 & 0.4116 & 0.3611 & 0.4638 \\
The third group & 0.3884 & 0.4517 & 0.4628 & 0.4326 \\
The fourth group & 0.4371 & 0.4103 & 0.4215 & 0.4597 \\
The fifth group & 0.4296 & 0.4908 & 0.4318 & 0.4257 \\
The Sixth group & 0.3915 & 0.5121 & 0.4459 & 0.4118 \\
\hline
\end{tabular}

\section{Design of BP network}

We use a three-layer BP network to predict the shooting percentage. There is one neuron in the input layer, 25 neurons in the hidden layer and 1 neuron in the output layer, which represents the predicted 
value of the shooting percentage. The activated functions of the hidden and output layers are Sigmoid and purelin functions respectively. Using the first four data in the table above it can predict the next data. Therefore, the input nodes are 4 and the output node is 1 . The function of nodes is the S-type function.

$$
f(x)=\frac{1}{1+e^{-x}}
$$

\section{Process of the Rolling Prediction Approach}

The rolling prediction of shooting percentage is the predictiong of the value in a future time based on a group of historic data. And then the predicted data will be seen as the historic data and make further prediction. In this cycle, the future values can be predicted step-by-step.

Network Training. We use the 1 st statistical competition data as the input of the network, and the 2-5th technical statistical data is set as the ideal output of the network, consisting of the training sample to train the network until the strong generalization network occurs.

Network Prediction. We set the 1st data in the table as the input of the network. The 1st data in the 2nd group was outputted by the network. Then the entire output is set as the new input, acquiring the 2 nd data in the 2 nd group. Following this rolling regulation, we can predict that the normalized basketball shooting percentage of the manteam in the next stage of the competition is 0.6486 and the anti-normalized value is 0.4601 .

\section{Summary}

The two features of the BP neural network algorithm can help us resolve the problems and difficulties of uncertainty in sports. The first one is that BP neural network algorithm can learn and store plenty of input/output reflection relationship without knowing the mathematical equations. The second one is that BP neural network algorithm has a very good self-adaption and self-organization capacity. Therefore, BP neural network algorithm can be used as an approach for predicting the scores in sport competitions. Meanwhile, the use of neural network toolbox in Matlab can predict the scores conveniently, improving the efficiency and accuracy of the modeling process.

\section{References}

[1] Mal. R, Mal. D, K. Differentiating between good credits and bad credits using neuro-fuzzy systems, Artificial Intelligence and Information Technology. 136(2002) 190-211.

[2] J F. Wang, B Zhang, Study on New Adaptive Complex Equalizers Con-structde by Radial Basis Function Neural Networks, Systems Engineering and Electronics. 25(2003) 848-859.

[3] Ismailov, Vugar E, On the approximation by neural networks with bounded number of neurons in hidden layers, Journal of mathematical analysis and applications. 417(2014) 963-969.

[4] F. Chen, H. Li, Z. Xu, S. Hou, D. Yang. User-friendly optimization approach of fed-batch fermentation conditions for the production of iturin A using artificial neural networks and support vector machine, Electron. J. Biotechnol. (2015).

[5] Turner B L, Kasperson R E, Matson P A, et al. A frame work for vulnerability analysis in sustainability science, PNAS. 100( 2003 ) $8074-8079$. 